\title{
Fibrodysplasia Ossificans Progressiva
}

National Cancer Institute

\section{Source}

National Cancer Institute. Fibrodysplasia Ossificans Progressiva. NCI Thesaurus. Code C3040.

A condition in which there is progressive heterotopic bone formation of the tendons and muscles. 